\title{
Communication Disorders and Speech Technology
}

\author{
Elmar Nöth ${ }^{1}$, Stefan Steidl ${ }^{1}$, and Maria Schuster ${ }^{2}$ \\ 1 University of Erlangen-Nuremberg, Chair of Pattern Recognition, Erlangen, Germany \\ noetheinformatik-uni-erlangen. de \\ http: //www5. informatik-uni-erlangen. de \\ 2 EuromedClinic, Fürth, Germany
}

\begin{abstract}
In this talk we will give an overview of the different kinds of communication disorders. We will concentrate on communication disorders related to language and speech (i.e., not look at disorders like blindness or deafness). Speech and language disorders can range from simple sound substitution to the inability to understand or use language. Thus, a disorder may affect one or several linguistic levels: A patient with an articulation disorder cannot correctly produce speech sounds (phonemes) because of imprecise placement, timing, pressure, speed, or flow of movement of the lips, tongue, or throat. His speech may be acoustically unintelligible, yet the syntactic, semantic, and pragmatic level are not affected. With other pathologies, e.g. Wernicke-aphasia, the acoustics of the speech signal might be intelligible, yet the patient is - due to mixup of words (semantic paraphasia) or sounds (phonematic paraphasia) - unintelligible.

We will look at what linguistic knowledge has to be modeled in order to analyze different pathologies with speech technology, how difficult the task is, and how speech technology is able to support the speech therapist for the tasks diagnosis, therapy control, comparison of therapies, and screening.
\end{abstract}

